\section{Mapping of soil layers using artificial neural network (case study of Babol, northern Iran)}

\author{
A J Choobbasti, F Farrokhzad, S Rahim Mashaie, P H Azar
}

Over the last few years, artificial neural networks (ANNs) have been used successfully for modelling all aspects of geotechnical engineering problems. ANNs are a form of artificial intelligence which attempt to mimic the function of the human brain and nervous system. ANNs are well suited to model the complex behaviour of most geotechnical engineering problems. The purpose of this paper was to assess the layering of subsurface soil using ANNs. Assessing the structure of soil layers on a site, depending on the extent of the study area, requires drilling several boreholes and performing several tests which demand considerable time and money. Increasing the knowledge of soil layer properties between boreholes leads to improved understanding of the near-surface geology. ANNs learn from data examples presented to them in order to capture the subtle functional data relationships, even if the underlying relationships are unknown or the physical meaning is difficult to explain. This paper focuses on the information gathered from the boreholes in a range of 40 square kilometres of Babol City in the north of Iran. The data was collected and classified in order to determine the characteristics of the soil layers. To later classify the different layers at different depths and to determine the thickness of each layer at a specified depth, multi-layer neural networks were trained separately. To quantify the neural network performance in estimating the changes of soil layers, some data from the test boreholes was presented to the network for the first time, and the results of neural networks were compared with actual data obtained from site investigations. The results show a high degree of accuracy in prediction by ANN models.

\section{INTRODUCTION}

During the last few decades researchers have studied the mechanical properties of soil, but the behaviour of soil is still not well known. Therefore, common methods such as mathematical and experimental methods use simplified hypotheses to solve multivariable geotechnical problems (Farrokhzad \& Choobbasti 2010). After reviewing these approaches, it can be said that these methods cannot describe the complex behaviour of soil. A substitution method is essential in which effective parameters are considered simultaneously, and generalisation capability and direct learning of experimental data should also be included (by considering errors). Neural networks have been utilised as a successful alternative method in recent years to solve many geotechnical engineering problems (Ghorbani et al 2012). Research in the field of neural networks has therefore been continuing due to the power of such tools in solving complex problems.

Different boreholes need to be drilled and many experiments performed to identify soil layer structures (Reinoso 2002). This process is very expensive and time-consuming (Alarcón et al 2004).
Increasing the reliability in interpolating soil layer structures and properties in interboreholes (i.e. distances among boreholes) improves the quality of the geotechnical evaluation and decreases the cost, and finally facilitates the proper planning of constructions (Aki 1993). ANN (as an intelligent system) uses some specific capabilities of data processing in the human brain, such as learning, data generalisation, handling the missing data and parallel processing, which were not available in previous systems. Many researchers studied ANN application in geotechnical engineering. Arel (2012) used the artificial neural network model to predict the complex soil of adapazari (Turkey), based on cone penetration test results. Penumadu and Zhao (1999) represented the deviator stress and volume change behaviour of varying types of sand and gravel for drained condition using artificial neural network. Farrokhzad et al (2012) proposed an ANN model to predict landslide risk. Ellis et al (1995) designed an ANN for sand and grain size distribution and stress history. Turk et al (2001) used ANN for modelling soil behaviour in uniaxial strain conditions. It should be mentioned that ANNs

\section{TECHNICAL PAPER}

\section{JOURNAL OF THE SOUTH AFRICAN INSTITUTION OF CIVIL ENGINEERING}

Vol 57 No 1, March 2015, Pages 59-66, Paper 1133

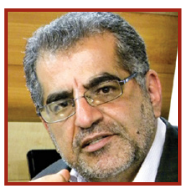

DRASSKARCHOOBBASTI is associate professor in the Department of Civil Engineering at Babol University of Technology, Iran. He obtained a BSC in Civil Engineering from Gilan University, Iran, in 1988, and a PhD in Civil Engineering from the University of UMIST, United Kingdom, in 1997. Among other positions, Dr Choobbasti served as head of the Department of Civil Engineering at Babol (2000-2004), dean of the Babol Noshirvani Engineering and Technical Complex (2005-2007) and president of Babol Noshirvani University of Technology (2009-2014). His research interests include soil liquefaction, numerical modelling and stone columns.

Contact details:

PO Box 484, Mazandaran, 47148 - 71167, Iran

T: +989113115904

E: asskar@nit.ac.ir

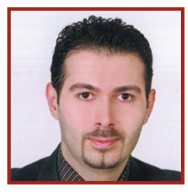

DR FARZAD FARROKHZAD is assisstant professo in the Department of Civil Engineering at the Mazandaran Institute of Technology, Iran. He obtained a BSc in Civil Engineering from Babol University of Technology, Iran, in 2005, and a PhD in Civil Engineering from the same university. He is head of the Department of Civil and Geotechnical Engineering at the Mazandaran Institute of Technology, Iran. He also serves as editor of the World Journal of Engineering Science, online editorial manager of AJGS (Springer), and editor of the International Journal of Civil and Structural Engineering. His research interests include liquefaction, site response analysis and soil dynamics.

\section{Contact details:}

Mazandaran Institute of Technology

PO Box 744, Mazandaran, 47148 - 71167, Iran

T: +989113123050

E:farzadfarokhzad@yahoo.com

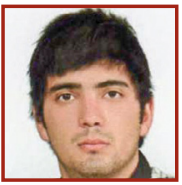

RAHIM MASHAIE obtained a BSc in Civil

Engineering from Azad University, Iran, in 2008, and an MSc in Geotechnical Engineering from the Mazandaran Institute of Technology, Iran, in 2013.

\section{Contact details:}

Mazandaran Institute of Technology

PO Box 744, Mazandaran, 47148 - 71167, Iran

T: +98 9113925689

E: sm_ramkat@yahoo.com

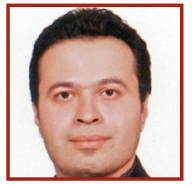

PEDRAM AZAR obtained his BSC in Civil Engineering from Babol University of Technology in 2004, and an MSc in Civil Engineering from the same university in 2006

\section{Contact details:}

Mazandaran Institute of Technology

PO Box 484, Mazandaran, 47148 - 71167, Iran

T: +989109101990

E:pedramix@gmail.com

Keywords: soil layering, artificial neural network, geotechnical investigation, Babol City 


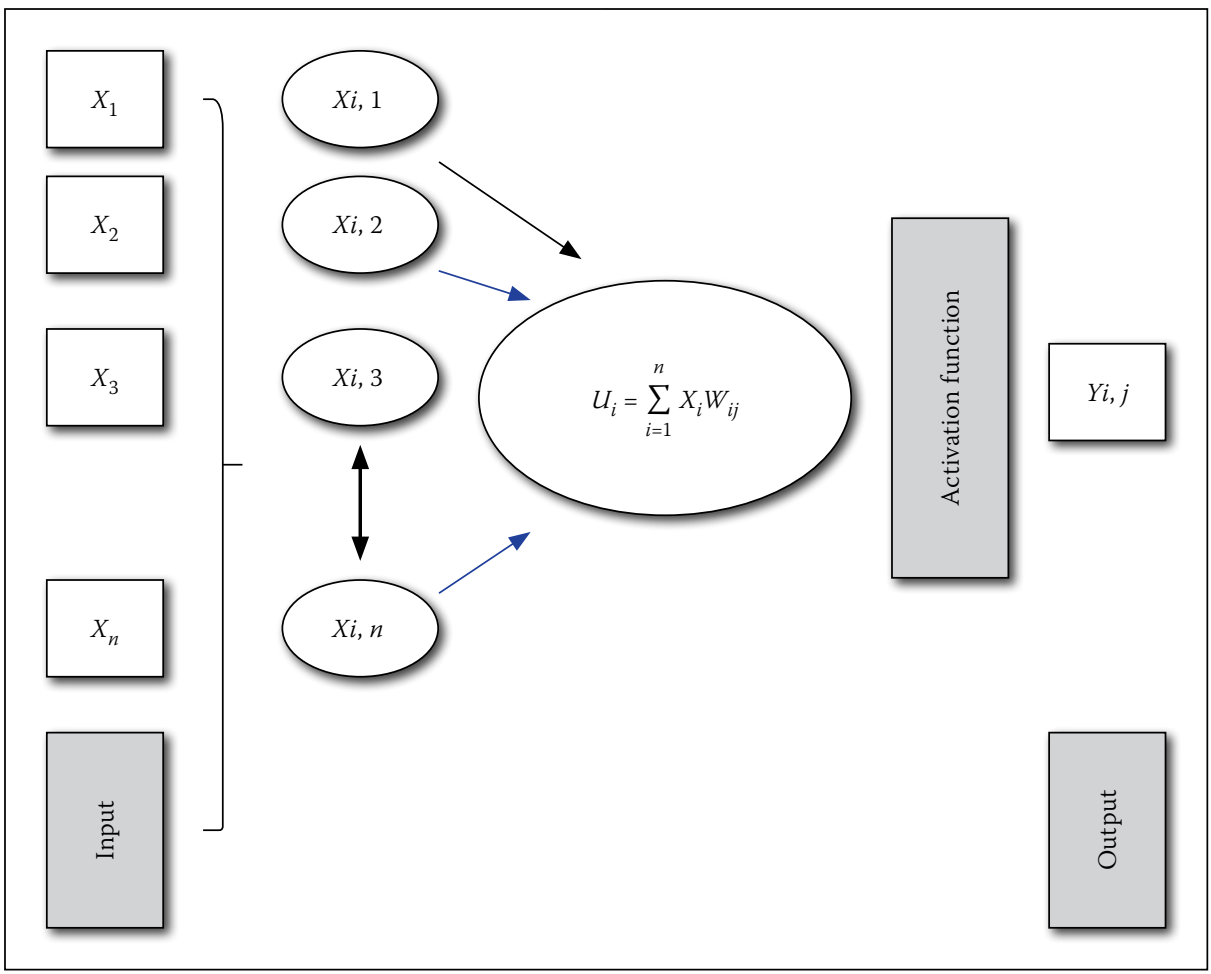

Figure 1 The architecture of an Artificial Neural Network

Table 1 Different categories for ANN input section based on soil type

\begin{tabular}{|l|l|l|l|l|l|l|l|l|l|}
\hline \multicolumn{3}{|c|}{ Clay } & \multicolumn{3}{c|}{ Silt } & \multicolumn{4}{c|}{ Sand } \\
\hline C & CL & CH & M & ML & MH & SM & SC & SW & SP \\
\hline
\end{tabular}

model the non-linear relation between the input parameters and the related outputs without considering the predefined mathematical relations (Farrokhzad et al 2012; Chavez-Garcia et al 2000; Choobbbasti et al 2009; Farrokhzad \& Choobbasti 2008).

\section{ARTIFICIAL NEURAL NETWORKS}

In recent years there has been a trend towards using computerised tools which are similar to biological nervous systems. These tools, which are known as "artificial neural networks", are found in almost all branches of engineering science and are being used increasingly. Their application in civil engineering dates from the late 1980s. Now a wide range of different applications exists, including process optimisation, the calculated value equivalent to vehicle axis load, performing and manufacturing process modelling, prediction of earthquake risk, and estimating implementing and manufacturing costs (Farrokhzad \& Choobbasti 2010).

The tendency towards using neural networks has increased due to a variety of factors, most important of which is the use of specific properties of information processing in the brain which are beyond the reach of conventional programming techniques - learning and generalising power, the possibility of proposing solutions for issues where the input might have some errors, the possibility of calculating related time responses for issues that have changing conditions, rapid data processing, and creating computers which have the possibility of working with high-volume calculations to provide training for neural networks.

\section{NEURON MODEL}

A neuron is a unit essential processor in a neural network. Figure 1 shows a neuron model which is the fundamental principle for designing artificial neural networks. A neuron $i$ can be written mathematically as in the following equation:

$\mathrm{Y}_{\mathrm{i}}=$ Activation Function $\left(U_{i}+b_{i}\right)$

$U_{i}=\sum_{i=1}^{n} X_{i} W_{i j}$

Where $x_{1}, x_{2} \ldots x_{n}$ are the input values; $w_{i 1}$, $w_{i 2} \ldots w_{i m}$ are the synaptic weights; $U_{i}$ is the line collector; $b_{i}$ Bios; $n(i)$ is the stimulating function and $y_{n}$ is the output neuron. The role of Bios $b_{i}$ involves a related conversion which is dependent on the output $u_{i}$ linear sum. There are three essential components in the model (Figure 1).
STUDY AREA AND GEOTECHNICAL INVESTIGATION

The geology, especially the tectonic style of Iran, is highly influenced by the development and history of the Tethyan region. The tectonic events, which occurred around the Iranian Plate margins, are related to rifting processes of Gondwana and the subsequent collision with the Arabian plate from the west-southwest. Adjoining fault-separated areas and tectonical units are the Alborz and Kopeh-Dagh regions to the north, the Makran and Zagros ranges to the west and south, and the east Iran ranges, which border this terrain to the east.

The collected information used in this research includes more than 45 boreholes drilled in the study area. Due to the large number of boreholes and the considerable changes in the soil layers observed during reviewing and studying the geological structure, an area of 40 square kilometres that has the highest density of data and similarity in the geological structure was selected at Babol. Among the raw data taken from the 45 boreholes in the selected range, 30 boreholes could be used in this study, ranging from $10 \mathrm{~m}$ to $21 \mathrm{~m}$ depths. Their dispersion is shown in Figure 2 (Choobbasti et al 2012; Farrokhzad et al 2012).

\section{MATERIAL AND METHODS}

Different methods for multi-layer neural network training have been invented, three of which are summarised below.

- Back propagation method: In the back propagation method, after selecting small random values for weight, an input vector is used and calculating is done layer by layer, and the output calculated. A neuron output from the output layer is reduced in order to determine the error. The degree of error in the derivative function, which is calculated for stimulation of these neurons in layer $k$, is multiplied. This error is used to adjust the network weights in the next step (Farrokhzad et al 2011b).

- Quick propagation method: This method uses the variables as a group, while in the back propagation method the weight changes after every application of the training data. In this method the mean surface slope error after applying all the data will be calculated, then the weights will be corrected. For this reason, in the quick propagation method the random sorting of data would not be useful. The scientific basis for the quick propagation method is the assumption that the level of error in each point (based on the position) is a contribution, and if the 
above-mentioned assumption is correct, after a small number of repeatings, the answer can easily be reached. Obviously, this assumption is not always true, but even if it is close to reality, the algorithm would rapidly be converging (Farrokhzad et al 2011c).

- Marquardt-Levenberg method: The Marquardt-Levenberg method (abbreviated as ML) can be used for training the network with a neuron in the output layer; the goal is to minimise the square sum of the nonlinear objective function. This method is based on Gauss-Newton's equations method. Based on the MarquardtLevenberg method, three separate multilayer networks were considered to solve the present problem. For each of the three selected networks in the first case, one or two hidden layers were considered; then, by applying the trial and error method for the number of middle-layer neurons, the useful method for network training and effective parameters in training (such as the learning rate, the momentum and the number of repeated steps - epoch which are necessary to achieve optimal performance) were determined.

Each training method yielded different results. In this case, performance was defined as the root-mean-squared error (RMSE) of the output patterns produced by the network on the testing data set. Each of the aforementioned supervised learning algorithms was evaluated. Back propagation is the best known training algorithm for neural networks and has the best performance (Farrokhzad et al 2011a).

\section{INPUT AND OUTPUT PARAMETERS}

In this research an ANN software package was used. It is based on MATLAB toolbox (MATLAB 8.0 and Statistics Toolbox 8.1). To train the ANN, the authors needed a set of known input and output pairs. The available input-output pairs are usually divided into two sets. The learning or training set is used to determine the connection weights in the networks. The testing set is used to measure the performance of the neural network after training.

As noted, considering the available data the decision was made to use data from the soil layers, so their classification results were obtained. The coordinates and depth $(x, y, z)$ were selected as input parameters. The related category responding to the input parameters, which will be described in the next paragraph, was introduced as the output of the ANN model. The soil type in the Unified Soil Classification System (USCS) was determined through the grain

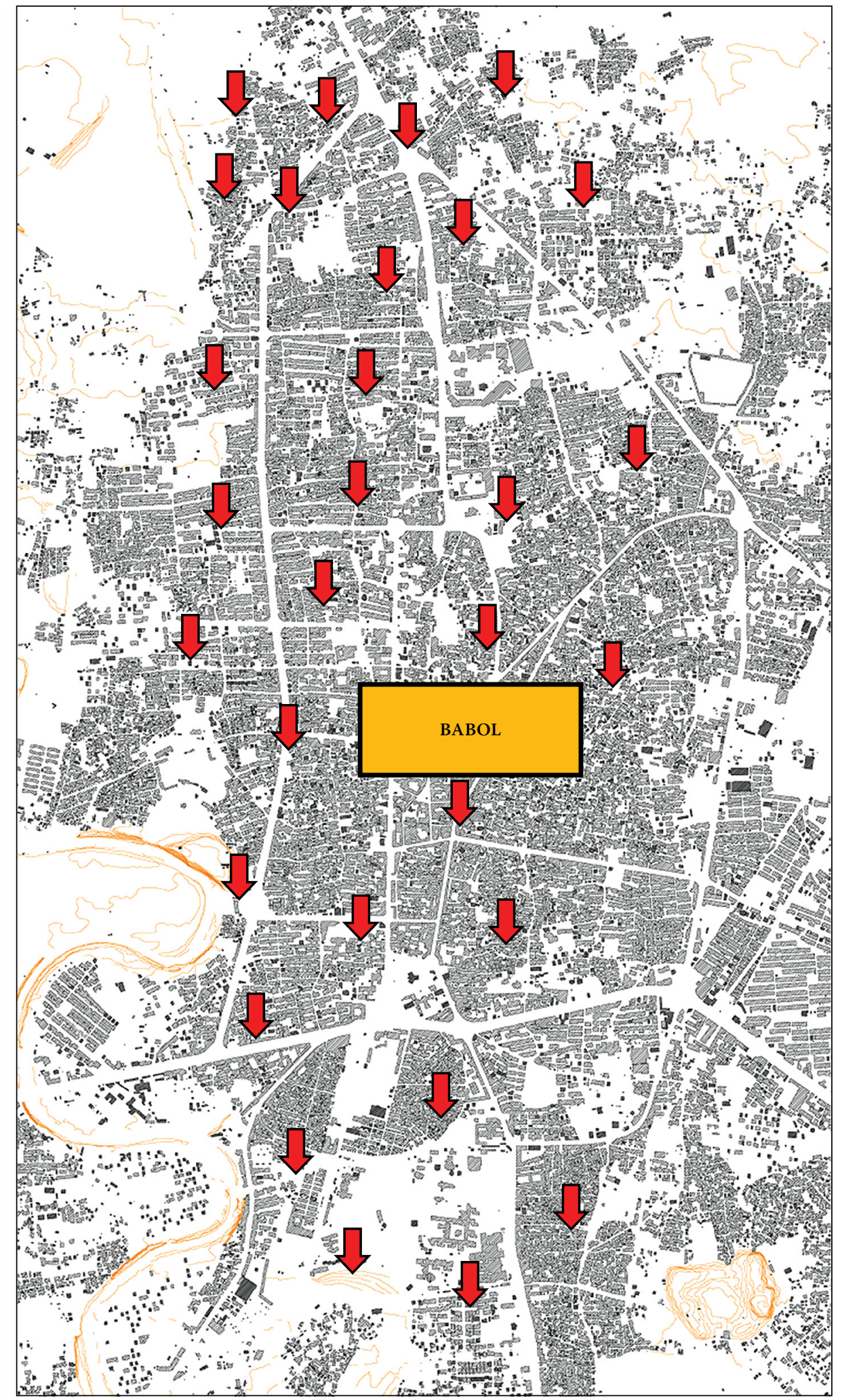

Figure $\mathbf{2}$ Location of some boreholes in the western part of Babol

size of samples (Kramer 1996). Soils from the Babol City study area were classified into ten groups (Table 1). In this way, symbols would be allocated to each designated group. In this research, $75 \%$ of the data was used for training and $25 \%$ for testing. A sample of a borehole log in the study area is shown in Table 2.

The RMSE values shown in Table 3 are based on different algorithms with one hidden layer. To obtain the proper neuron numbers in the hidden layer, a network with constant characteristics was considered; then, by changing the effective parameters of the network and assigning different values for neurons, the results could be compared to determine the optimum number of neurons. As mentioned above, RMSE was our measure to determine the neuron number. Back-propagation neural networks with $2,3,4,5,6,7,8,9,10$ neurons in hidden layers were trained. In Table 4, RMSE measurements for different neurons are shown. The minimum RMSE belongs to a 
Table 2 A sample of borehole log obtained form site investigation (central part of Babol)

\begin{tabular}{|c|c|c|c|c|c|c|c|c|c|c|c|c|c|c|c|}
\hline \multirow{2}{*}{ 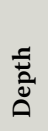 } & \multirow{2}{*}{$\underset{w}{\tilde{W}}$} & \multirow{2}{*}{$\begin{array}{l}\text { Field description } \\
\text { of soils }\end{array}$} & \multirow{2}{*}{ 灾 } & \multicolumn{3}{|c|}{$\begin{array}{c}\text { Ground water level } \\
1.4 \mathrm{~m}\end{array}$} & \multirow{2}{*}{ W\% } & \multirow{2}{*}{$Y_{d}$} & \multicolumn{2}{|c|}{ D.S (3) } & \multicolumn{2}{|c|}{ T.T (4) } & \multicolumn{3}{|c|}{ Con. T (5) } \\
\hline & & & & LL & PL & PI & & & $\mathrm{C}$ & $\Phi$ & $\mathrm{C}$ & $\Phi$ & Cc & Cs & Pc \\
\hline 1 & \multirow{2}{*}{ ML } & \multirow{2}{*}{ Medium plastic silt } & \multirow{2}{*}{7} & \multirow{2}{*}{36.97} & \multirow{2}{*}{29.60} & \multirow{2}{*}{7.37} & \multirow{2}{*}{28.99} & \multirow{2}{*}{1.50} & & & & & & & \\
\hline 2 & & & & & & & & & & & & & & & \\
\hline 3 & \multirow{2}{*}{ ML } & \multirow{2}{*}{ Medium plastic silt } & \multirow{2}{*}{7} & \multirow{2}{*}{42.34} & \multirow{2}{*}{29.71} & \multirow{2}{*}{12.63} & \multirow{2}{*}{31.11} & \multirow{2}{*}{1.47} & \multirow{2}{*}{0.11} & \multirow{2}{*}{35.34} & & & \multirow{2}{*}{0.145} & 0.043 & 100 \\
\hline 4 & & & & & & & & & & & & & & & \\
\hline 5 & $\mathrm{MH}$ & High-plastic silt & 13 & 54.17 & 31.79 & 22.38 & 30.70 & 1.48 & & & & & & & \\
\hline 6 & & & & & & & & & & & & & & & \\
\hline 7 & & & & & & & & & & & & & & & \\
\hline 8 & $\mathrm{CL}$ & Medium plastic clay & 6 & 45.35 & 25.48 & 19.87 & 27.50 & 0.78 & & & & & 0.127 & 0.049 & 1.50 \\
\hline 9 & & & & & & & & & & & & & & & \\
\hline 10 & ML & Non-plastic silt & 19 & 26.96 & 26.96 & 0.00 & 28.56 & 1.42 & & & & & & & \\
\hline 11 & & & & & & & & & & & & & & & \\
\hline 12 & & & & & & & & & & & & & & & \\
\hline 13 & ML & Medium plastic silt & 10 & 42.41 & 26.79 & 15.62 & 36.02 & 1.34 & & & & & & & \\
\hline 14 & & & & & & & & & & & & & & & \\
\hline 15 & $\mathrm{CL}$ & Non-plastic clay & 10 & 39.07 & 23.60 & 15.47 & 23.92 & 1.47 & & & & & & & \\
\hline 16 & & & & & & & & & & & & & & & \\
\hline 17 & & & & & & & & & & & & & & & \\
\hline 18 & ML & Very stiff plastic silt & 21 & 41.73 & 26.75 & 14.98 & 34.11 & 1.44 & & & & & & & \\
\hline 19 & & & & & & & & & & & & & & & \\
\hline 20 & $\mathrm{MH}$ & High-plastic silt & 29 & 50.07 & 33.40 & 16.67 & 25.12 & 1.54 & & & & & & & \\
\hline 21 & ML & Very stiff plastic silt & 30 & & & & & & & & & & & & \\
\hline & & ithology Description & & Unit we & $\mathrm{t}(\mathrm{gr} / \mathrm{cr}$ & & Direct s & $\operatorname{ar}(\mathrm{gr} / \mathrm{c}$ & & Triax & 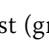 & & Con & ation & \\
\hline
\end{tabular}

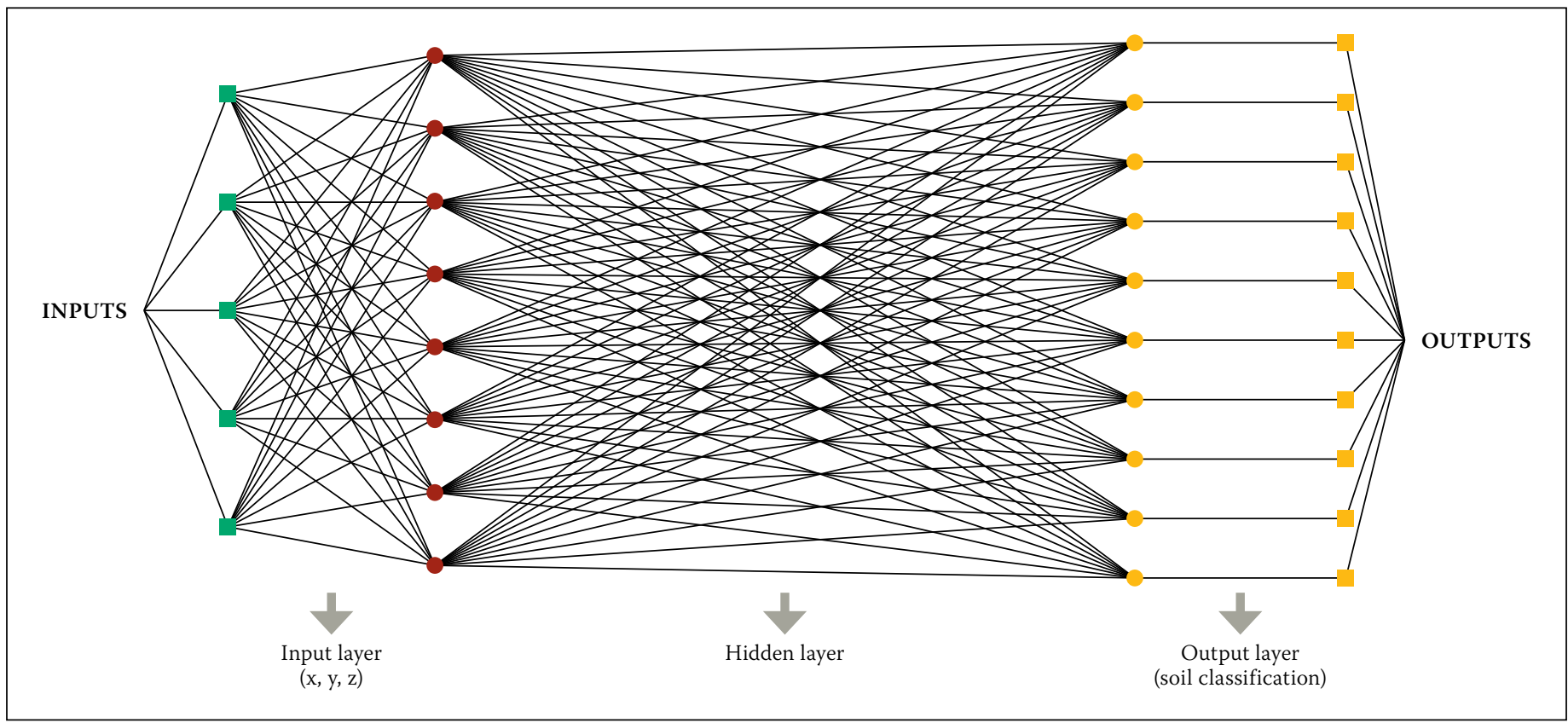

Figure 3 The network structure

Table 3 Results of learning algorithms

\begin{tabular}{|c|c|c|c|}
\hline $\begin{array}{c}\text { Supervised learning } \\
\text { algorithms }\end{array}$ & Quick propagation & Back propagation & Marquardt-Levenberg \\
\hline RMSE (\%) & 21.30 & 17.41 & 24.70 \\
\hline
\end{tabular}

network with seven neurons and momentum $=0.2$, learning rate $=0.3$, and epoch $=500$. The suitable ANN model for the mentioned problem was selected based on a lower RMSE value. 


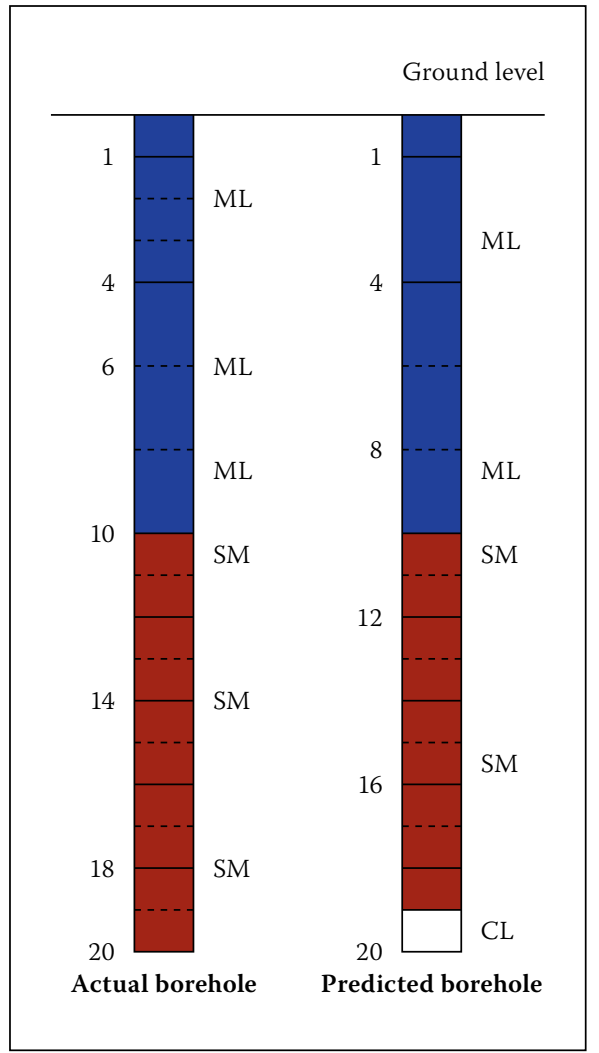

Figure 4 Comparison of actual and predicted layers in a borehole

\section{RESULTS}

Figure 3 shows the architecture of the designed network to predict soil layers in a borehole at the site. As can be seen, Figure 3 describes the mechanism of the proposed neural networks for predicting the class of soil layer. Given $(x, y, z)$ coordination, the network recognises the class of layer.

Figure 4 shows a sample log of a borehole that was predicted with ANN against the actual data. It can be said that the results of ANN demonstrate a high level of accuracy.

Figure 5 shows an example of the designed networks. In order to evaluate the classification strategy, there are three different performance measurements, the first being correctly classified instances for total accuracy in predicting instances. The second measures are true positive rate (TPR) and false positive rate (FPR). While TPR is a positive measure to assess the performance of the network, the FPR is a negative measure in this manner. The performance of the network can be exactly evaluated for each soil layer in a borehole. Confusion matrix is the last alternative which can assist with evaluating the classification strategy. Rows and columns of the matrix indicate the type of soil layers. Elements in the main diagonal indicate the accuracy of the network. The higher the values in the main diagonal, the more accurate the network will be. For example, in Figure 5 one misclassification

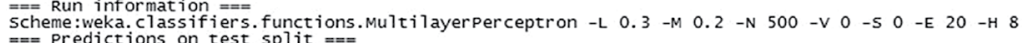

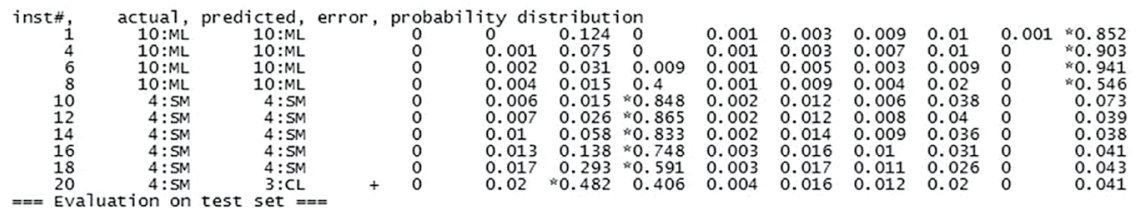
$==E$ Evaluation on test set $===$
$== \pm$ Summary $== \pm$ correctly classified Instances Kappa statistic Mean absolute error
Root mean squared err Relative absolute error
Root relative squared error
Total Number of $\begin{array}{ll}\text { Total Number of Instances } & 41.7374 \%\end{array}$

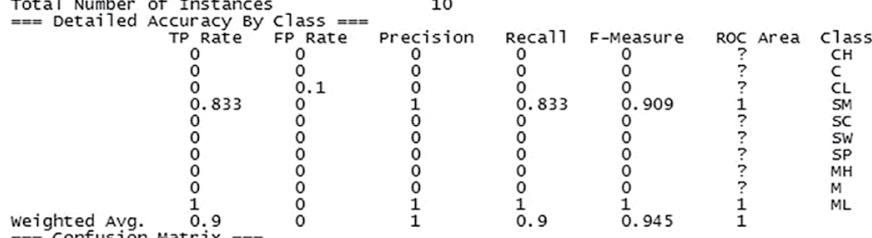

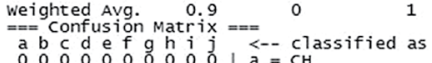

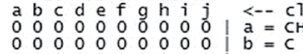

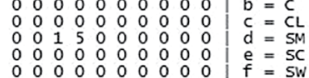

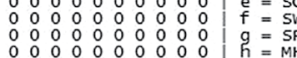

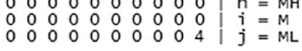

\section{Figure 5 A sample of Artificial Neural Network toolbox and modelling}

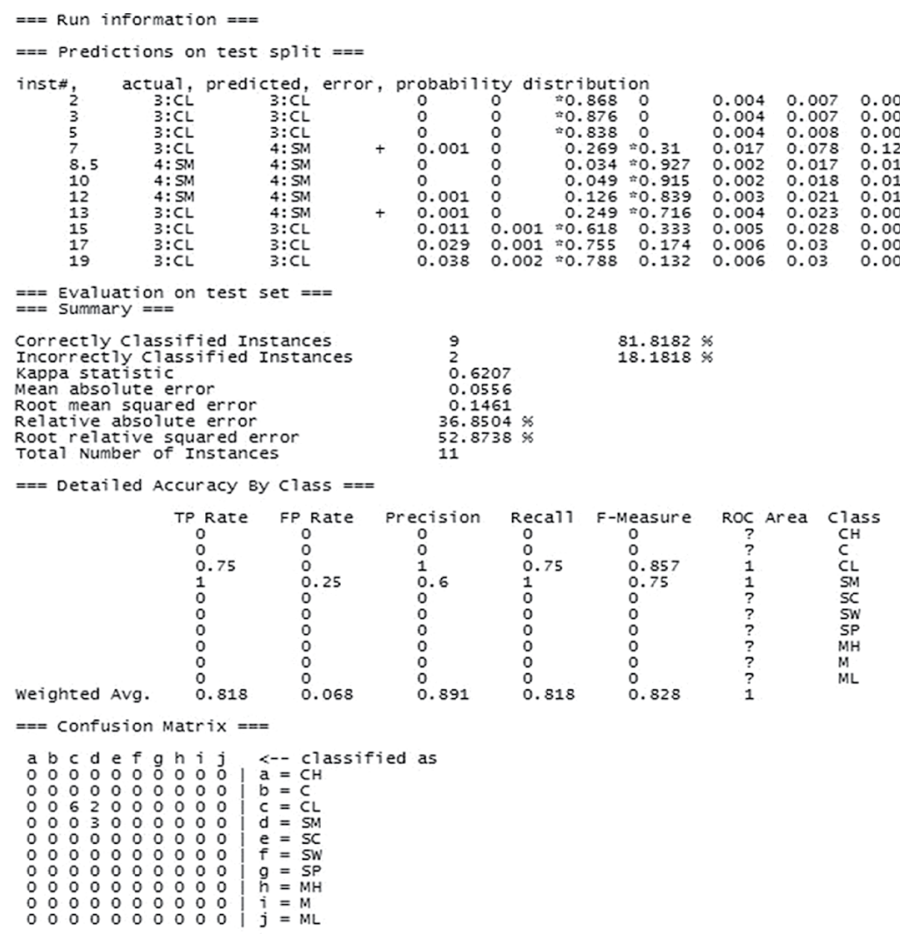

Figure 6 A sample of ANN test algorithm

Table 4 The RMSE values for different neurons in hidden layers

\begin{tabular}{|l|c|c|c|c|c|c|c|c|c|}
\hline $\begin{array}{c}\text { Number of neurons } \\
\text { in hidden layers }\end{array}$ & $\mathbf{2}$ & $\mathbf{3}$ & $\mathbf{4}$ & $\mathbf{5}$ & $\mathbf{6}$ & $\mathbf{7}$ & $\mathbf{8}$ & $\mathbf{9}$ & $\mathbf{1 0}$ \\
\hline RMSE (\%) & 16.46 & 15.04 & 16.77 & 15.30 & 16.59 & 14.70 & 14.88 & 14.97 & 14.79 \\
\hline
\end{tabular}

occurred in predicting SM soil; therefore one element out of the main diagonal is non-zero.

Figure 6 shows the performance of the network in predicting soil layers in one of the boreholes located in the study area. As can be observed, and also demonstrated in the first part of the figure, the network shows over $80 \%$ accuracy in most cases to predict two different soil profiles, ML and $\mathrm{SM}$, which exist in this borehole. There is only one failure in this particular observation and it is related to the last soil layer in which the network mistakenly predicted 
the SM soil as the CL. With regard to the failure in the last layer, it is notable that the network allocated a 40.6 probability to the existence of SM soil and a 42.8 probability to the existence of $\mathrm{CL}$ soil, so the reason that this layer is mistakenly named a CL soil layer is due to the higher percentage of probability allocated to CL. It can be inferred that the variability of the percentage addressed to these two soils is less than $10 \%$ and supports the reasonable performance of designed networks in predicting requested elements.

TPR and FPR are two indexes describing independently the percentage correct and incorrect classifications per soil type in one borehole. For example, when the index of TPR for SM soil in this borehole is described as $83.3 \%$, it means the network has predicted five out of every six SM soil layers in this borehole correctly, and the average of five for six items is equal to $83.3 \%$; and when the FPR index for SM soil is equal to 0 , that means none of the soil layers in this borehole has been incorrectly predicted as SM soil.

Figure 7 demonstrates 18 boreholes in the western part of Babol City. The type and amount of soil in each borehole are selected according to Table 1 categories. A multi-layer perceptron (MLP) is used to predict the depth and class of soil layers. Its training algorithm is based on back propagation, and its learning type is also supervised. ANN achieved up to $90 \%$ accuracy in predicting soil layers.

In order to test the ANN model, seven boreholes from the test categories were selected (Figures 8 and 9). The accuracy of the model is shown in Table 5. The accuracy values are measured by comparison of actual data and prediction of ANN, as in Figure 4. The accuracy rate is about $90 \%$, as presented in Table 5.

\section{CONCLUSION}

In this paper the authors constructed and trained an ANN model to predict the soil layers of Babol City (between boreholes). The data was gathered from the boreholes in a range of 40 square kilometres of Babol City in the north of Iran. A multi-layer neural network was trained to classify the different layers of soil at different depths. It should be mentioned that the trained ANN model can determine the thickness of each layer at a specified depth. To ensure network performance in estimating the changes of soil layers, data from the test boreholes was presented to the network for the first time and the results of neural networks were compared with actual data obtained from site investigations. The results show about

Table 5 Accuracy and performance of the models

\begin{tabular}{|l|c|c|c|c|c|c|c|}
\hline Number of bore hole & $\mathbf{1 5}$ & $\mathbf{3}$ & $\mathbf{9}$ & $\mathbf{2}$ & $\mathbf{6}$ & $\mathbf{1 7}$ & $\mathbf{4}$ \\
\hline Accuracy & $91 \%$ & $88 \%$ & $83 \%$ & $91.5 \%$ & $90.7 \%$ & $89 \%$ & $92.8 \%$ \\
\hline
\end{tabular}

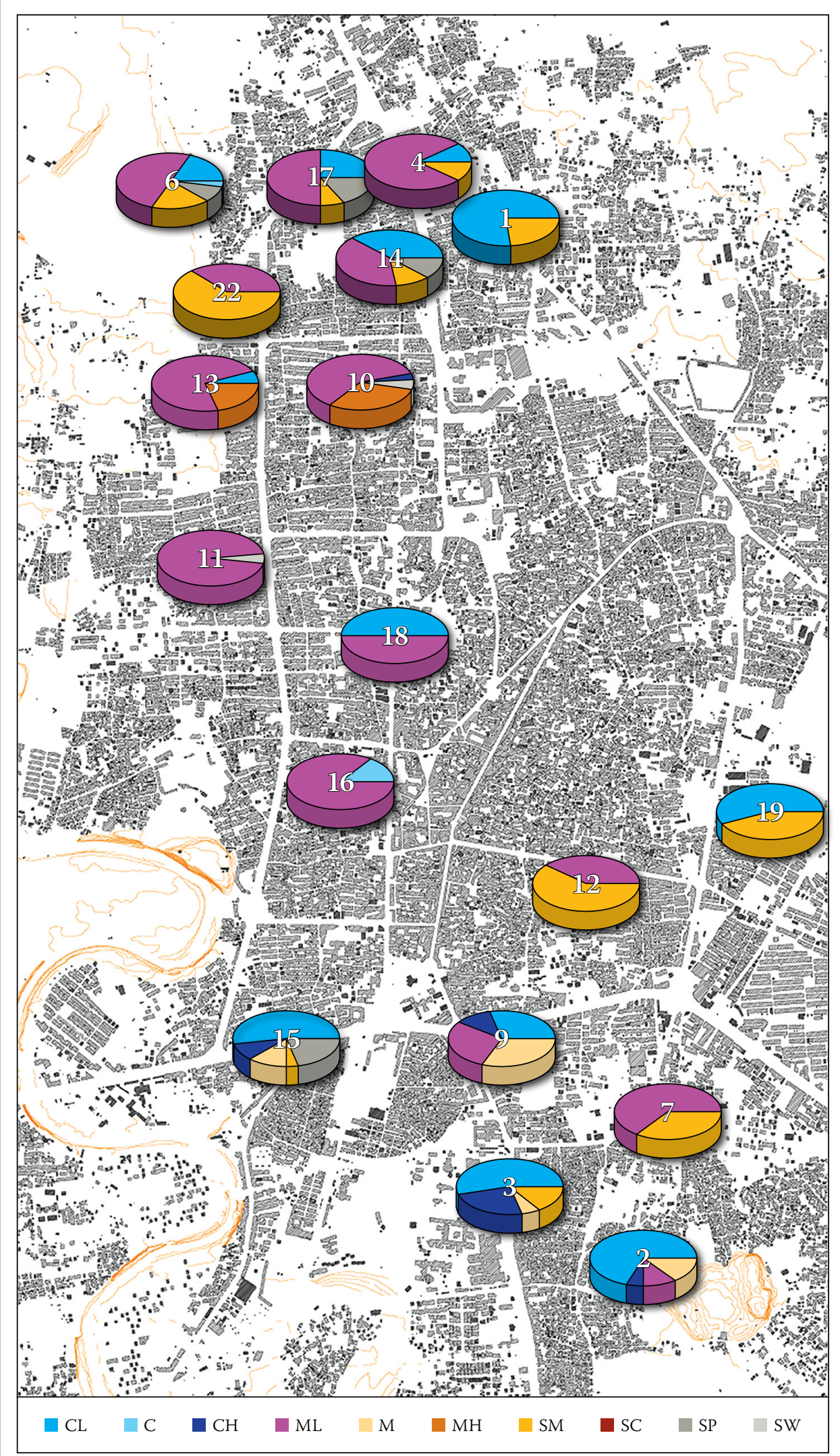

\section{Figure 7 Type and amount of soil in each borehole}

$90 \%$ accuracy in prediction by the trained ANN model.

As a result, extracted information could be used for site investigation in the first step of each geotechnical project. It is notable that the input instances should be in the range of the trained ones which help to improve the performance of ANNs. In other 


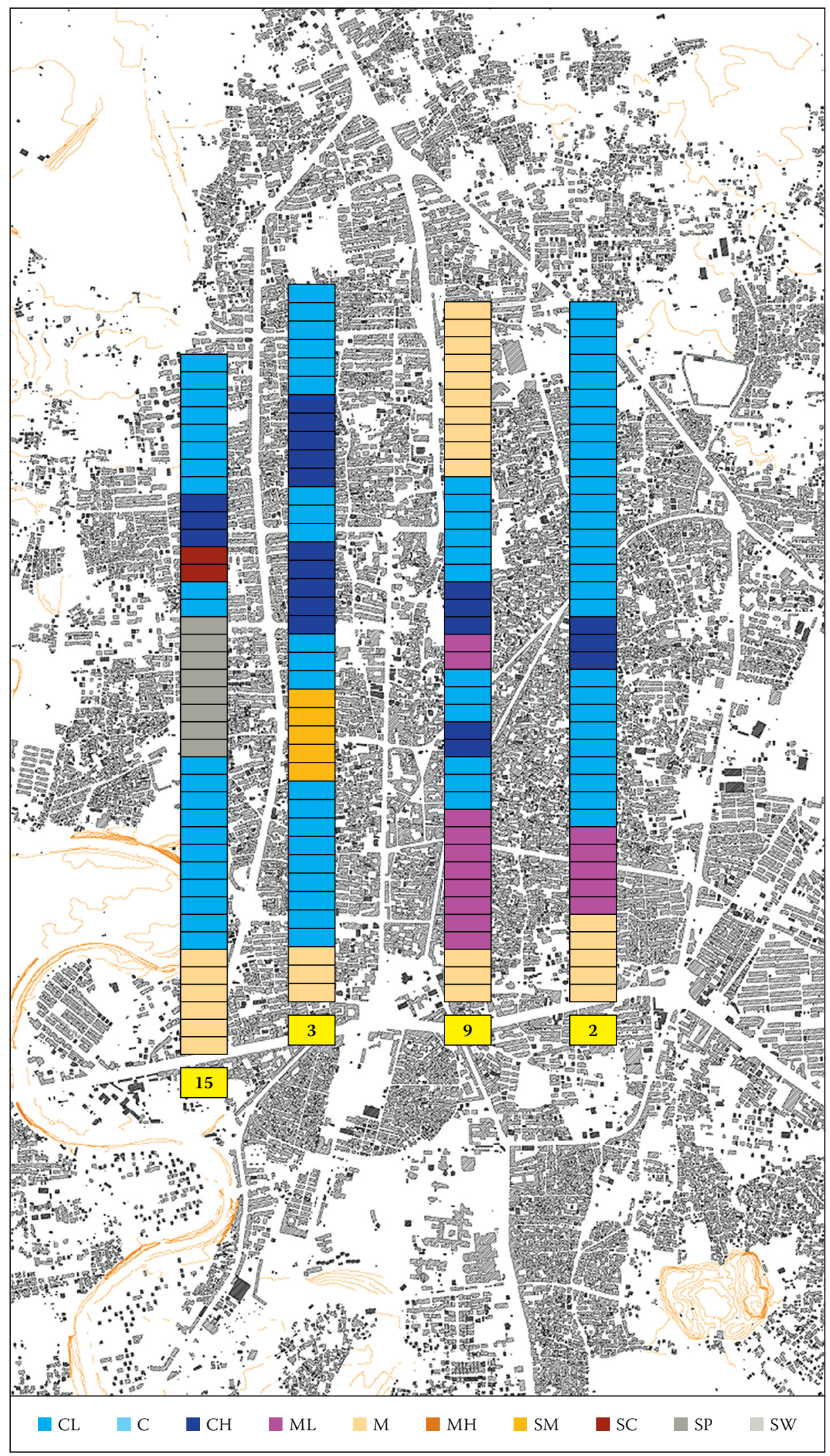

Figure 8 Sample of prediction in the eastern part of Babol

words, an introduced ANN model is capable of interpolating the input-output pairs; future research will define the accuracy of ANN models in extrapolation of data sets. Regarding the accuracy of the designed ANN model it can be said that this model is capable of predicting the subsurface soil layers in a study area. Therefore the need to drill more boreholes is reduced, which could lead to saving money and time.
Arel, E 2012. Predicting the spatial distribution of soil profile in Adapazari/Turkey by artificial neural network using CPT data. Computers \& Geosciences, 43: 90-100.

Chavez-Garcia, F J, Raptakis, D, Makra, K \& Pitilakis, K 2000. Site effects at Euroseistest-II. Results from 2D numerical modeling and comparison with observations. Soil Dynamics and Earthquake Engineering, 19: 23-39.

Choobbbasti, A J, Barari, A \& Farrokhzad, F 2009. Prediction of slope stability using artificial neural network (Case study: Noabad, Mazandaran, Iran). Arabian Journal of Geosciences, 2: 311-319.

Choobbasti, A J, Shooshpash, E \& Farrokhzad, F 2012.

3-D modeling of soil's unsaturated depth using artificial neural network (Case study of Babol). Unsaturated Soils: Research and Applications, 2: 317-324.

Ellis, G W, Yao, C, Zhao, R \& Penumadu, D 1995. Stress-strain modeling of sands using artificial neural networks. Journal of Geotechnical Engineering, 121: 429-435.

Farrokhzad, F \& Choobbasti, A J 2008. Prediction of subsurface soil layers using artificial neural network. Proceedings, International Conference on Numerical Computation in Geotechnical Engineering, Skikda, Algeria.

Farrokhzad, F \& Choobbasti, A J 2010. Artificial neural network model for prediction of liquefaction potential in soil deposits. Proceedings, Fifth International Conference on Recent Advances in Geotechnical Earthquake Engineering and Soil Dynamics, San Diego, CA, US.

Farrokhzad, F, Barari, A, Choobbasti, A J \& Ibsen, L B 2011a. Neural network-based model for landslide susceptibility and soil longitudinal profile analyses: Two case studies. Journal of African Earth Sciences, 61: 349-357.

Farrokhzad, F, Barari, A, Ibsen, L B \& Choobbasti, A J 2011b. Predicting subsurface soil layering and landslide risk with artificial neural networks: A case study from Iran. Geologica Carpathica, 62: 477-485.

Farrokhzad, F, Choobbasti, A J, \& Barari, A 2011c. Determination of liquefaction potential using artificial neural networks. Gradevinar, 63: 837-845 Farrokhzad, F, Choobbasti, AJ \& Barari, A 2012. Liquefaction microzonation of Babol city using artificial neural network. Journal of King Saud University, 24: 89-100.

Ghorbani, A, Jafarian, Y \& Maghsoudi, M S 2012. Estimating shear wave velocity of soil deposits using polynomial neural networks: Application to liquefaction, computers and geosciences. Computers \& Geosciences, 44: 86-94.

Kramer, S 1996. Geotechnical Earthquake Engineering, Englewood Cliffs, NJ, US: Prentice-Hall.

MATLAB 8.0 and Statistics Toolbox 8.1. Natick, MA, US: The MathWorks, Inc.

Penumadu, D \& Zhao, R 1999. Triaxial compression behavior of sand and gravel using artificial neural networks (ANN). Computers and Geotechnics, 24: 207-230. 
Reinoso, E 2002. Scattering of seismic waves.

Applications to the Mexico City valley (Advances in Earthquake Engineering). London: WIT Press.

Turk, G, Logar, J \& Majes, B 2001. Modelling soil behavior in uniaxial strain conditions by neural networks. Advances in Engineering Software, 10: $805-812$.

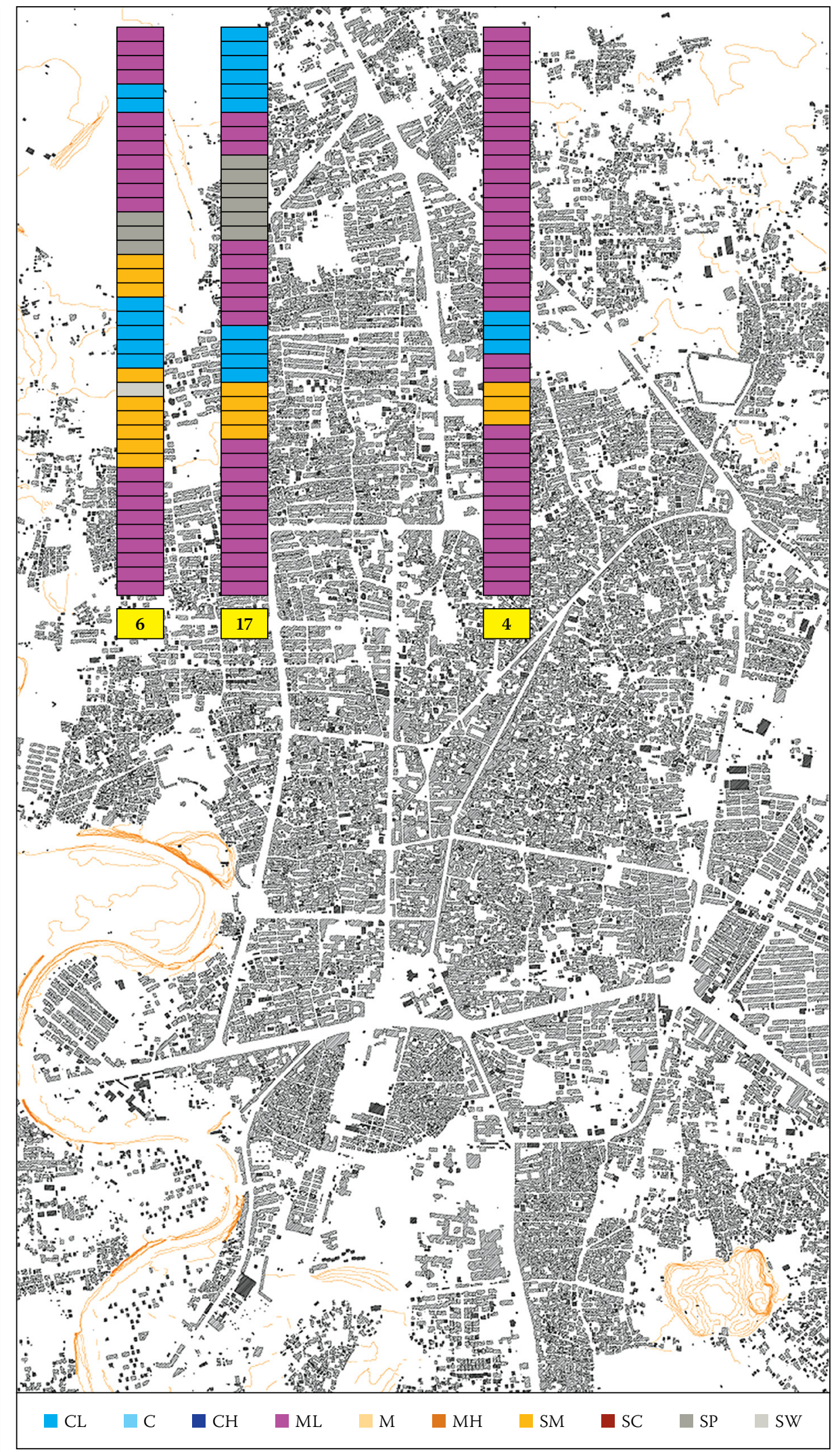

Figure 9 Sample of prediction in the western part of Babol 